\title{
THE SIMPLE GROUP OF ORDER 2520
}

\author{
BY G. A. MILLER
}

\author{
Extract from a letter to F. N. Cole
}

If a second simple group of order $7 ! / 2$ existed it could not be represented as a primitive group whose degree is less than twenty-one since all these primitive groups have been determined. The number of its subgroups of order 7 would be 120 , for the only other divisor of $7 ! / 2$ which is of the form $1+7 k$ and greater than 20 is 36 . It is easy to prove that such a simple group could not involve exactly 36 subgroups of order 7 , as follows.

If a simple group of order $7 ! / 2$ contained exactly 36 subgroups of order 7 , it could be represented as a transitive group $G$ on 36 letters representing the permutations of these 36 subgroups. Its subgroup $G_{1}$ composed of all its substitutions omitting one letter would be of order 70 . It would therefore involve a cyclic subgroup of order 35 which would be regular, since the substitutions of order 7 would be regular. The subgroup $G_{1}$ could not be dihedral, since it could not involve negative substitutions. For the same reason the substitutions of order 2 could not transform the substitutions of order 5 into themselves and the substitutions of order 7 into their inverses. If these substitutions of order 2 could transform the substitutions of order 7 into themselves and the substitutions of order 5 into their inverses, $G_{1}$ would involve 5 conjugates of order 2 . But this is impossible, since 36.5 is not divisible by 8 .

Having proved that if the group in question existed it would contain 120 subgroups of order 7 , we proceed to consider its subgroups of order 9 . The number of these subgroups would be divisible by 35 . In fact, if an operator of order 5 or an operator of order 7 could transform a subgroup of order 9 into itself it would be commutative with each of its operators. It was proved above that an operator of order 7 cannot be 
commutative with every operator of a subgroup of order 9 . If an operator of order 5 were commutative with every operator of such a subgroup, the number of the subgroups of order 5 would be 56 . Hence the group in question could be represented as a transitive group $G$ of degree 56 , in which $G_{1}$ would be an abelian group of order 45 involving substitutions of degree 55 and order 5 . The subgroup of order 9 contained in $G_{1}$ could not have a transitive constituent of degree 9 , since 8.56 is not divisible by 11 . For a similar reason it could not have a substitution of degree 30 . Hence $G$ could not involve exactly 56 subgroups of order 5 .

Since the number of subgroups of order 9 would be divisible by 35 , this number would be either 70 or 280 . In the latter case, the group in question could be represented as a transitive group $G$ of degree 280 , in which $G_{1}$ would be of degree 279 and of order 9 . If all the substitutions besides the identity, of $G_{1}$ were of degree $279, G$ would be of class 279 and hence could not be simple. Hence $G_{1}$ would involve at least three transitive constituents of degree 3 . If a substitution of $G_{1}$ were of degree 276 , it would be invariant under 4 and only 4 subgroups of order 9, and hence under a subgroup of order 36 which would contain an invariant subgroup of order 4 . Hence the group under consideration could be represented as a transitive group $G$ of degree 70 in which the $G_{1}$ of order 36 contains four subgroups of order 9 .

The invariant subgroup of order 4 contained in this $G_{1}$ would be invariant under a larger group since it would be invariant under a group of order 8. This larger group would contain more than 4 subgroups of order 9 and the number of these subgroups would be divisible by 8 . As this is impossible, it has been proved that if $G$ contains 280 subgroups of order 9 the $G_{1}$ of the transitive group according to which they are transformed cannot contain a substitution of degree 276 .

If this $G_{1}$ contained a substitution of degree 273 , there would be a subgroup of order 63 which would involve an invariant subgroup of order 7, but this is contrary to the result obtained above. If it contained a substitution of degree 270 , there 
would be a subgroup of order 90 which would have a quotient group of order 30 . As the latter could not contain ten subgroups of order 3, this is impossible. Since no substitution of this $G_{1}$ could be of a lower degree than 270 , it has been proved that the number of subgroups of order 9 contained in $G$ is exactly 70, and that $G$ can therefore be represented as a transitive group of degree 70 representing the transformations of its subgroups of order 9 . The $G_{1}$ of this $G$ is therefore of degree 69 and of order 36 and contains a single subgroup of order 9 .

The substitutions in this subgroup of order 9 cannot all be of degree 69 , since 9 does not divide 69 . Hence this group of order 9 contains at least one substitution whose degree does not exceed 66. If it contains no substitution besides the identity of lower degree, it has two and only two transitive constituents of degree 3 . In this case $G_{1}$ must have a transitive constituent of degree 6 , since it cannot involve an invariant subgroup of order 3 and of degree less than 69 . Moreover, the transitive constituent of degree 6 must be of order 36 , as otherwise $G_{1}$ would involve an invariant substitution of order 2 whose degree could not exceed 36 . The four subgroups of order 9 which are transformed into themselves by a substitution of order 3 and of degree 66 must therefore generate a group of order 36 in which this substitution is invariant, since a subgroup of order 18 in a transitive group of degree 6 and order 36 does not contain an invariant substitution of degree 3 . It was proved above that this subgroup of order 36 contains an invariant subgroup of order 4 involving three conjugate substitutions of order 2 .

As this subgroup of order 36 could have only 9 operators in common with $G_{1}$, its invariant subgroup of order 3 would be transformed into itself by 9 operators of the latter subgroup which are not found in the former subgroup of order 36 . Hence we may confine our attention to the case when this invariant subgroup of order 3 would be transformed into itself by exactly 72 operators of $G$, and when $G$ would involve a single set of 35 conjugate subgroups of order 3 . Hence $G$ 
may be supposed to be represented as a primitive group of degree 35 and its $G_{1}$ has an invariant subgroup of order 3 whose substitutions are invariant under a $G_{36}$ and are of degree 30. This $G_{36}$ contains 26 substitutions of order 3 of which 10 are of degree 30 and 16 of degree 33, while its three substitutions of order 2 are of degree 28 since it has three transitive constituents, of degrees 18,12, and 4, respectively. All of these conclusions follow from the properties of the subgroup of order 36 considered in the preceding paragraph. Each of the subgroups of order 9 has three regular constituents of order 9 .

The $G_{1}$ of order 72 has also three transitive constituents, one of which is the symmetric group of degree 4 . It involves 21 substitutions of order 2 and of degree 28 and 18 of order 4 and of degree 34 . As all the non-invariant non-cyclic subgroups of order 4 contained in this $G_{1}$ are conjugate, this $G_{1}$ is completely determined. Its generalized dihedral subgroups of order 18 are therefore also determined. Hence the group of order 36 which contains such a dihedral group is also determined, and there is no such group besides the known simple $G$, which can be represented on 7 letters. It should be noted that there is a substitution of order 2 which is commutative with every substitution of the transitive constituent of degree 18 and involves only the letters of this constituent, but is not found therein. This permutes its two regular constituents of order 9 which appear in the dihedral group of order 18 noted above and it is on this account that the group of order 36 in question is completely determined. This group contains a constituent of degree 2 and together with $G_{1}$ generates $G$.

It has now been proved that if there were a second simple group of order $7 ! / 2, G_{1}$ would involve substitutions whose degree would be less than 66 . As the subgroup of order 9 in $G_{1}$ could not contain exactly five transitive constituents of degree 3 it would have to involve at least 8 such constituents. If $G_{1}$ contained a substitution of order 3 and of degree 63 , the seven subgroups of order 9 which would be transformed into themselves by this substitution would generate a group in which this substitution is invariant, and hence this group would con- 
tain exactly 7 subgroups of order 9 . Its order would therefore be 63,126 , or 252 . This is impossible as each of these groups would involve only one subgroup of order 7 since each of its subgroups of order 7 would be transformed into itself by at least 21 substitutions.

It remains only to consider the case when $G_{1}$ would contain a substitution of order 3 and of degree 60 without involving such a substitution of degree 63 . The order of the group formed by all the substitutions of $G$ which would be commutative with this substitution of order 3 would be 90 . This group of order 90 would transform its ten subgroups of order 9 according to a transitive group of order 30 and of degree 10 . Since this transitive group does not exist, ${ }^{*}$ we have arrived at nothing but contradictions by assuming the existence of a second simple group of order $7 ! / 2$ and hence such a group is actually proved to be non-existent.

The University of Illinois.

\section{A THEOREM OF OSCILLATION}

BY W. E. MILNE

In an investigation of the oscillations of aerial bombs a need was found for the following proposition. Both the theorem and its proof are modelled after a similar theorem and proof by Osgood. $\dagger$

THEOREM. Let $\varphi(t)$ be positive, continuous, monotonically increasing, and bounded in the interval $T \leqq t<\infty$, and let $m$ and $M$ be two positive constants such that $m<\varphi(t)<M$ for $t>T$. Let $f(y)$ be an odd, monotonically increasing function, satisfying the Lipschitz condition

$$
\left|f\left(y_{1}\right)-f\left(y_{2}\right)\right|<K\left|y_{1}-y_{2}\right|, \quad K>0,
$$

in an interval $-a \leqq y \leqq+a, a>0$. Let $y$ be a solution of the differential equation

$$
\frac{d^{2} y}{d t^{2}}+\varphi(t) f(y)=0
$$

* Cf. F. N. Cole, Quarterly Journal, vol. 27 (1895), p. 40.

$\dagger$ This Bulletin, vol. 25 (1919), pp. 216-221. 